\title{
Predictors of Relapse after Inpatient Opioid Detoxification during 1-Year Follow-Up
}

\author{
Harsh Chalana, Tanu Kundal, Varun Gupta, and Amandeep Singh Malhari \\ Department of Psychiatry, Sri Guru Ram Dass Institute of Medical Sciences and Research, Amritsar 143501, India \\ Correspondence should be addressed to Harsh Chalana; harsh_chalana@yahoo.co.in
}

Received 7 April 2016; Revised 31 July 2016; Accepted 24 August 2016

Academic Editor: Angela L. Stotts

Copyright ( 2016 Harsh Chalana et al. This is an open access article distributed under the Creative Commons Attribution License, which permits unrestricted use, distribution, and reproduction in any medium, provided the original work is properly cited.

\begin{abstract}
Introduction. Relapse rate after opioid detoxification is very high. We studied the possibility that predetoxification patient characteristics might predict relapse at follow-up and thus conducted this 1-year follow-up study to assess the predictors of relapse after inpatient opioid detoxification. Materials and Methods. We conducted this study in our tertiary care institute in India over two-year time period (1 Jan 2014 to 31 Dec 2015). Out of 581 patients admitted, 466 patients were considered for study. Results and Discussion. No significant difference was found between relapsed and nonrelapsed patients regarding sociodemographic profile; however substance abuse pattern and forensic history showed significant differences. Relapsed patients abused greater amount and used injections more commonly, as compared to nonrelapsed group. Longer duration of abuse was also a significant risk factor. Patients with past attempt of opioid detoxification and family history (parental or first degree) of alcohol abuse had decreased possibility of maintaining remission during 1-year follow-up. Relapsed patients were found to abuse their spouse or parents. Conclusion. Our study compared profiles of relapsed and nonrelapsed patients after inpatient detoxification and concluded predictors of relapse during 1-year follow-up period. Early identification of predictors of relapse and hence high risk patients might be helpful in designing more effective and focused treatment plan.
\end{abstract}

\section{Introduction}

Relapse rate after opioid detoxification ranges from 72 to $88 \%$ after $12-36$ months, despite multidisciplinary endeavors, though a six-month controlled study has shown lower relapse rate (32-70\%) [1,2]. Improvement in this rate can be done by a better understanding of pretreatment risk factors, including patient characteristics, associated with relapse after inpatient detoxification.

Early relapse after inpatient detoxification has been found to be significantly predicted by younger age, greater heroin use prior to treatment, history of injecting, and failure to enter aftercare [3]. Similarly, categories of relapse precipitant have been identified as cognition, mood, external, withdrawal, interpersonal, leaving a protected environment, drug availability, drug-related cues, craving, priming, and social pressure, in a follow-up study of 78 opiates abusers after successful opioid detoxification [4]. Abstinence has also been found to be significantly associated with completion of the 6-week inpatient treatment program and attendance at outpatient aftercare and negatively associated with a family history of substance misuse [5].

In an outpatient detoxification program, interpersonal factors, drug-related cues such as regularly meeting other drug users and being offered drugs, and persistent negative mood states have been found to be associated with relapse into opiate use [6]. Recent developments in cognitive neuroscience point to neurocognitive measures (i.e., brain-imaging measures during cognitive-task performance) as potential predictors of relapse over and above the information gained from self-report measures such as craving [7].

However, in contrast to findings in a long term study, same author earlier studied medium term follow-up outcomes after inpatient treatment of opioid dependence and concluded that patient preadmission characteristics account for a very small proportion of the variance in outcomes $[3,5]$. Another 2.5-year follow-up study also found preadmission client characteristics unsuccessful in predicting achievement of abstinence [8]. 
We studied the possibility that predetoxification patient characteristics might predict relapse at follow-up and thus conducted this 1-year follow-up study to assess the predictors of relapse after inpatient opioid detoxification.

\section{Materials and Methods}

We conducted this study in Department of Psychiatry (Deaddiction unit), Shri Guru Ram Das Institute of Medical Sciences and Research, Vallah, Amritsar, Punjab, India, over two-year time period (1 Jan 2014 to 31 Dec 2015), after permission from Institutional Ethics Committee. Inclusion criteria was any patient with a diagnosis of opioid dependence (as per ICD-10 criteria), admitted for detoxification in the deaddiction unit from 1 Jan 2014 to 31 Dec 2014 and consenting to participate in study. Exclusion criteria included refusal to consent, comorbid other drug addictions (except tobacco), comorbid other psychiatric or significant medical ailments, age $<18$ years, and known history of any adverse reaction with Naltrexone.

A total of 581 patients were admitted from 1 Jan 2014 to 31 Dec 2014 out of which 115 subjects met defined exclusion criteria or did not meet inclusion criteria. Remaining 466 patients were considered for study. A detailed history was taken and sociodemographic performa (Appendix) was completed for every patient. Average stay of subjects for detoxification varied from two to four weeks depending on withdrawal signs and symptoms. Inpatient detoxification was done as per standard protocol and medications were gradually tapered off to stop after 1-3 weeks except Quetiapine. Tab Quetiapine was used for affective symptoms as per need. After being abstinent from opioids for a minimum of 5-7 days, all patients were discharged on Tab Naltrexone $50 \mathrm{mg}$ o.d. with or without Tab Quetiapine 50-200 mg/d, with regular weekly visits in outpatient unit, for next 1 year.

At least one attendant/caregiver was identified for every patient during inpatient stay, which was mostly a close family member and would stay along with patient. They were made responsible for supervising daily medication at home and were advised to make note if they suspect their patient for any substance abuse. Urine for drug abuse was done randomly to monitor relapse. A total of 2512 random samples were taken, out of which 103 were positive for opioids and they were considered as relapse. None of the patients were positive for any other substance abuse (except tobacco). Patients and their attendants were interviewed regarding relapse, which was defined as abuse of any substance, except tobacco. Alcohol abuse was also not reported by any patient or his attendant.

Adherence therapy of all patients was done at every visit by trained psychologist. Relapsed patients were compared with nonrelapsed patients with respect to their sociodemographic variables as per performa. Patients, who were lost to follow-up, were considered as relapse. Their last observations were carried forward to calculate the final data, rather than considering only the completed subjects, to avoid the bias. We tried to contact them telephonically to ask about their reason for loss to follow-up.

Relapsed and nonrelapsed groups were compared across the variables using chi-square test. A multivariate logistic regression analysis was conducted to identify variables that were independently associated with opiate abstinence. All the tests were two-tailed, and a value of $P<0.05$ was considered statistically significant.

\section{Results and Discussion}

3.1. Sociodemographic Profile. A total of 466 patients were included in our study during the study period (1 Jan 2014 to 31 Dec 2014) and followed up for next 1 year (till Dec 2015). All patients were male. Sociodemographic profile has been provided in Table 1. Majority was in the age range $20-40$ and had rural background. Most were married and employed with low income range. Education level was predominantly above matriculation. No significant difference was found between relapsed and nonrelapsed patients.

3.2. Drug Use Profile. Heroin was the most common substance of abuse in both groups as evident in Table 2. However, relapsed patients abused greater amount and used injections more commonly, as compared to nonrelapsed group. Longer duration of abuse was also a significant risk factor. Presence of craving at discharge from hospital after detoxification was significant in both groups and logistic regression showed that craving at discharge was significantly associated with relapse $(\beta=6.86, P=0.01)$. Patients with past attempt of opioid detoxification and family history (parental or first degree) of alcohol abuse had decreased possibility of maintaining remission during 1-year follow-up.

3.3. Forensic History Profile. As shown in Table 3, patients with history of police case and incarceration were found to be significantly associated with high risk of relapse. Relapsed patients were also found to verbally and physically abuse their spouse or parents. History of self-harm was not found to be a significant risk factor for relapse.

\section{Conclusion}

(1) Our study compared profiles of relapsed and nonrelapsed patients after inpatient detoxification and concluded predictors of relapse during 1-year follow-up period. High number of young males in both groups represent pattern of drug abuse in population, in general. No female getting admitted for detoxification in our study might be due to high stigma associated with substance abuse; however females have been reported in a significant number depending on city and geographical location, in other studies [9]. Young age has been found to have high risk of relapse after inpatient detoxification $[3,10]$ but our study did not show any relation of age with relapse. High rural percentage in whole sample shows local area representation, as our tertiary care institute is located in rural area and mostly caters surrounding rural population. A majority of patients in both groups were married and employed, which envisages need to involve family members in treatment process. However, low economic status of patients despite high cost of substances of abuse may suggest downward economic drift of substance abusers as 
TABLE 1: Sociodemographic profile.

\begin{tabular}{|c|c|c|c|c|}
\hline & Relapsed $(n=147)$ & Nonrelapsed $(n=319)$ & Chi-square value & Signific. \\
\hline \multicolumn{5}{|l|}{ Age } \\
\hline Below 20 years & $45(30.61 \%)$ & $70(21.94 \%)$ & \multirow{3}{*}{4.70} & \multirow{3}{*}{ Ns } \\
\hline $20-40$ years & $80(54.42 \%)$ & $195(61.13 \%)$ & & \\
\hline Above 40 & $22(14.50 \%)$ & $54(16.93 \%)$ & & \\
\hline \multicolumn{5}{|l|}{ Resid. status } \\
\hline Rural & $102(69.39 \%)$ & $223(69.90 \%)$ & \multirow{2}{*}{0.01} & \multirow{2}{*}{ Ns } \\
\hline Urban & $45(30.61 \%)$ & $96(30.09 \%)$ & & \\
\hline \multicolumn{5}{|l|}{ Marital status } \\
\hline Single & $41(27.89 \%)$ & $82(25.71 \%)$ & \multirow{3}{*}{0.25} & \multirow{3}{*}{ Ns } \\
\hline Married & $85(57.82 \%)$ & $190(59.56 \%)$ & & \\
\hline Divor./separated & $21(14.29 \%)$ & $47(14.73 \%)$ & & \\
\hline \multicolumn{5}{|l|}{ Empl. status } \\
\hline Employed & $61(41.50 \%)$ & $148(46.39 \%)$ & \multirow{3}{*}{3.20} & \multirow{3}{*}{ Ns } \\
\hline Unemployed & $56(38.10 \%)$ & $95(29.78 \%)$ & & \\
\hline Prev. employed & $30(20.40 \%)$ & $76(23.82 \%)$ & & \\
\hline \multicolumn{5}{|l|}{ Income (INR pm) } \\
\hline $0-<10000$ & 77 (52.38\%) & $165(51.72 \%)$ & \multirow{3}{*}{0.78} & \multirow{3}{*}{ Ns } \\
\hline $10000-<20000$ & $37(25.17 \%)$ & $91(28.52 \%)$ & & \\
\hline 20000-above & $33(22.45 \%)$ & $63(19.74 \%)$ & & \\
\hline \multicolumn{5}{|l|}{ Education } \\
\hline Illiterate & $28(19.05 \%)$ & 45 (14.11\%) & \multirow{3}{*}{3.06} & \multirow{3}{*}{ Ns } \\
\hline Up to matric. & $39(26.53 \%)$ & $79(24.76 \%)$ & & \\
\hline Above matric. & $80(54.42 \%)$ & $195(61.13 \%)$ & & \\
\hline
\end{tabular}

well as alternate source of money being used for buying substances. Education does not appear to act as a deterrent factor for substance abuse or preventing relapse; however economic literacy awareness might be more helpful. Our findings were similar to another study done in a tertiary centre in India, to assess predictors for treatment retention in a tertiary centre in India, where all subjects were males and majority of the sample was married, educated up to matriculation, and employed and belonged to the nuclear family and urban background. Higher socioeconomic status and having a family member with substance use were associated with higher chances of treatment retention [11].

(2) Greater amount of heroin use, longer duration, history of injecting, and $>3$ lifetime heroin-quit attempts have been found to be significant predictors of relapse $[3,12]$. Our study corroborates all these findings. We also found that heroin was the most preferred opioid of abuse among all patients, which might be due to its easy availability. Our patient base represents mainly Amritsar District, which due to its geographical location, being on border with neighboring country Pakistan, is more prone to be affected with smuggled heroin. Further, more than $1 \mathrm{gm}$ of heroin, injection use, and more than 3 years of use prior to detoxification were significantly associated with relapse within 1 year. Craving was found to be present in majority of patients at time of discharge after detoxification, but it did not predict relapse in our study, though compulsive use immediately prior to hospitalization has been found to be significant in another study [13]. We noted that 2 or more previous attempts for detoxification were significantly associated with relapse; however previous studies have shown both supportive and contradictory association [14, 15]. Further, past psychiatry history or admission (except substance abuse) was not found to be associated with relapse in our findings. We also noted significant history of alcohol abuse in parents and first-degree relatives of relapsed patients, similar to another follow-up study in which $19 \%$ patients reported history of parental alcohol misuse, but surprisingly opioid abuse history in family was not found to be associated with relapse in our study [5]. Inpatient treatment and regular follow-ups have also been found to be significantly associated with abstinence $[3,5,16]$. Our studies design ensured admission and regular follow-ups for all patients which might have been a reason for lower overall relapse rate as compared to other researches.

(3) We found police case and imprisonment history to be significant in relapsed cases as shown in Table 2. One reason of high relapse in these patients could be presence of antisocial traits which itself is a risk factor for substance abuse. However, client characteristics were not found to be associated with relapse in two different studies, one a 2.5-year follow-up study and the other was a medium term follow-up study $[5,8]$. One of the reasons for different results could be that, in 2.5-year follow-up study, analysis was done 2.5 years after detoxification. Another reason could be difference in culture and treatment setup. In our culture, patients without antisocial traits are better monitored by their family members while those with such traits may not be very compliant to their family values. 
TABle 2: Substance misuse.

\begin{tabular}{|c|c|c|c|c|}
\hline & Relapsed $(n=147)$ & Nonrelapsed $(n=319)$ & Chi-square & Level of sign. \\
\hline \multicolumn{5}{|c|}{ Substance misuse history } \\
\hline \multicolumn{5}{|l|}{ Principal opiate of use } \\
\hline Morphine & $10(6.80 \%)$ & $30(9.40 \%)$ & \multirow{7}{*}{5.15} & \multirow{7}{*}{ Ns } \\
\hline Poppy husk & $12(8.16 \%)$ & $31(9.71 \%)$ & & \\
\hline Heroin/smack & $70(47.61 \%)$ & $150(47.02 \%)$ & & \\
\hline Tramadol & $15(10.20 \%)$ & $20(6.26 \%)$ & & \\
\hline Dextropropoxyphene & $9(6.12 \%)$ & $29(9.09 \%)$ & & \\
\hline Diphenoxylate & $3(2.04 \%)$ & $9(2.82 \%)$ & & \\
\hline Combination of opiates & $28(19.04 \%)$ & $50(15.67 \%)$ & & \\
\hline \multicolumn{5}{|l|}{ Quantity (heroin per day) } \\
\hline Less than $0.5 \mathrm{gm}$ & $23(17.68 \%)$ & $110(34.48 \%)$ & \multirow{3}{*}{57.52} & \multirow{3}{*}{0.01} \\
\hline $0.5-1 \mathrm{gm}$ & $38(25.85 \%)$ & $136(42.63 \%)$ & & \\
\hline More than $1 \mathrm{gm}$ & $86(58.50 \%)$ & $73(22.88 \%)$ & & \\
\hline \multicolumn{5}{|l|}{ Predominant route } \\
\hline Chase/smoke & $4(2.72 \%)$ & $70(21.94 \%)$ & \multirow{4}{*}{43.85} & \multirow{4}{*}{0.01} \\
\hline Inject & $70(47.61 \%)$ & $90(28.21 \%)$ & & \\
\hline Oral & $28(19.04 \%)$ & $83(26.01 \%)$ & & \\
\hline Multiple & $45(30.61 \%)$ & $76(23.82 \%)$ & & \\
\hline \multicolumn{5}{|l|}{ Duration of abuse } \\
\hline$<1$ year & $10(6.80 \%)$ & $101(31.66 \%)$ & \multirow{3}{*}{48.85} & \multirow{3}{*}{0.01} \\
\hline $1-3$ years & $47(31.97 \%)$ & $118(36.99 \%)$ & & \\
\hline$>3$ years & $90(61.22 \%)$ & $100(31.34 \%)$ & & \\
\hline \multicolumn{5}{|l|}{ Craving at discharge } \\
\hline Yes & $123(83.67 \%)$ & $248(77.74 \%)$ & \multirow{2}{*}{2.18} & \multirow{2}{*}{ Ns } \\
\hline No & $24(16.32 \%)$ & $71(22.25 \%)$ & & \\
\hline \multicolumn{5}{|c|}{ Number of previous attempted opiate detoxifications } \\
\hline 0 & $45(30.61 \%)$ & $101(31.66 \%)$ & \multirow{3}{*}{49.55} & \multirow{3}{*}{0.01} \\
\hline 1 & $13(8.84 \%)$ & $118(36.99 \%)$ & & \\
\hline Two or more & $89(60.54 \%)$ & $100(31.34 \%)$ & & \\
\hline \multicolumn{5}{|c|}{ Past (nonaddiction) psychiatric history } \\
\hline Yes & $36(24.48 \%)$ & $102(31.975)$ & \multirow{2}{*}{2.71} & \multirow{2}{*}{ Ns } \\
\hline No & $111(75.51 \%)$ & $217(68.02 \%)$ & & \\
\hline \multicolumn{5}{|l|}{ Inpatient psy treatment } \\
\hline Yes & $9(6.12 \%)$ & $21(6.58 \%)$ & 0.03 & $\mathrm{Ns}$ \\
\hline No & $138(93.87 \%)$ & $298(93.41 \%)$ & 0.03 & Ns \\
\hline Parental alcohol misuse & & & & \\
\hline Yes & $88(59.86 \%)$ & $57(17.86 \%)$ & 82.80 & 0.01 \\
\hline No & $59(40.13 \%)$ & $262(82.13 \%)$ & 82.80 & 0.01 \\
\hline Parental opiate abuse & & & & \\
\hline Yes & $42(28.57 \%)$ & $109(6.80 \%)$ & 144 & Ns \\
\hline No & $105(71.42 \%)$ & $210(65.83 \%)$ & 1.44 & Ns \\
\hline First-degree relative alcoh & & & & \\
\hline Yes & $95(64.62 \%)$ & $74(23.19 \%)$ & 7472 & 001 \\
\hline No & $52(35.37 \%)$ & $245(76.80 \%)$ & $/ 4 . / 2$ & 0.01 \\
\hline First-degree relative opiat & & & & \\
\hline Yes & $55(37.41 \%)$ & $125(39.18 \%)$ & 013 & Ns \\
\hline No & $92(62.58 \%)$ & $194(60.47 \%)$ & 0.13 & Ns \\
\hline
\end{tabular}


TABLE 3: Forensic history.

\begin{tabular}{|c|c|c|c|c|}
\hline & Relapsed $(n=147)$ & Nonrelapsed $(n=319)$ & Chi-square & Level of sign. \\
\hline \multicolumn{5}{|c|}{ Forensic history } \\
\hline \multicolumn{5}{|c|}{ Police case registered } \\
\hline Yes & $24(16.32 \%)$ & $18(5.64 \%)$ & \multirow{2}{*}{14.01} & \multirow{2}{*}{0.01} \\
\hline No & $123(83.67 \%)$ & $301(94.35 \%)$ & & \\
\hline \multicolumn{5}{|c|}{ Imprisonment } \\
\hline Yes & $12(8.16 \%)$ & $7(2.19 \%)$ & \multirow{2}{*}{9.17} & \multirow{2}{*}{0.01} \\
\hline No & $135(91.83 \%)$ & $312(97.81 \%)$ & & \\
\hline \multicolumn{5}{|c|}{ History of verbal abuse } \\
\hline Yes & $100(68.02 \%)$ & $111(34.80 \%)$ & \multirow{2}{*}{44.85} & \multirow{2}{*}{0.01} \\
\hline No & $47(31.97 \%)$ & $208(65.20 \%)$ & & \\
\hline \multicolumn{5}{|c|}{ History of physical abuse } \\
\hline Yes & $65(44.22 \%)$ & $47(14.73 \%)$ & \multirow{2}{*}{47.91} & \multirow{2}{*}{0.01} \\
\hline No & $82(55.78 \%)$ & $272(85.27 \%)$ & & \\
\hline \multicolumn{5}{|c|}{ History of self-harm } \\
\hline Yes & $8(5.44 \%)$ & $20(6.27 \%)$ & \multirow{2}{*}{0.12} & \multirow{2}{*}{ Ns } \\
\hline No & $139(94.55 \%)$ & $299(93.73 \%)$ & & \\
\hline
\end{tabular}

Another reason for high relapse in the patients in our study could be habituation of offenses. Illegality is a major deterrent for opioid abuse but involvement in police cases and being in jail possibly habituates the offender, who finally does not find it as a deterrent anymore. Incarceration was found to be most common cause of dropout in a community based Indian study [9]. Involvement in legal issues has been reported as one of the major factors for treatment failure [17]. Our study also found history of verbal and physical abuse of spouse or parents as a significant risk factor for relapse. Positive family functioning and relationships have been reported to be significantly associated with improvement at follow-up $[18,19]$. Problem with spouse was found to be a precipitant factor in another follow-up study [20].

To conclude, early identification of predictors of relapse and hence high risk patients might be helpful in designing more effective and focused treatment plan. More research is needed to explore patient characteristics based on our study which may help to decrease recurrent admissions and hospital expenses.

\section{Limitations}

Relapse criteria relied on interview with patients and their accompanying attendants, rather than regular urine screening tests, although random tests were done.

\section{Appendix}

\section{Performa for Research Study}

Name:

Sex:

MRD No.:

Serial No.:
Socio-Demographic Profile

Age

Below 20 years

20-40 years

Above 40

Resid. Status

Rural

Urban

Marital Status

Single

Married

Divor./Separated

Empl. Status

Employed

Unemployed

Prev. employed

Income (INR pm)

$$
\begin{aligned}
& 0-<10000 \\
& 10000-<20000 \\
& 20000-\text { Above }
\end{aligned}
$$

Education

Illiterate

Up to Matric.

Above Matric. 
Substance Misuse

Principal opiate of use

Morphine

Poppy Husk

Heroin/ Smack

Tramadol

Dextropropoxyphene

Diphenoxylate

Combination of opiates

Quantity (heroin per day)

Less than $0.5 \mathrm{gm}$

0.5-1 gm

More than $1 \mathrm{gm}$

Predominant route

Chase/smoke

Inject

Oral

Multiple

Duration of abuse

$<1$ year

1-3 years

$>3$ years

Craving at Discharge

Yes

No

No. of previous attempted opiate detoxification

0

1

Two or more

Past (non-addiction) Psychiatric history

Yes

No

In-patient psy treatment

Yes

No

Parental Alcohol misuse

Yes

No
Parental opiate abuse

$$
\text { Yes }
$$$$
\text { No }
$$

First rel. alcohol abuse

$$
\begin{gathered}
\text { Yes } \\
\text { No }
\end{gathered}
$$

First relative opiate abuse

$$
\text { Yes }
$$$$
\text { No }
$$

Forensic History

Police Case Registered

$$
\begin{gathered}
\text { Yes } \\
\text { No }
\end{gathered}
$$

Imprisonment

Yes

No

History of verbal abuse

Yes

No

History of physical abuse

Yes

No

History of Self Harm

Yes

No

\section{Competing Interests}

The authors declare that they have no competing interests.

\section{References}

[1] H. Chalana, J. K. Sachdeva, T. Kundal, A. S. Malhari, and R. Choudhary, "A double blind, placebo controlled, randomised study comparing Quetiapine with placebo, along with oral Naltrexone, in the treatment of opioid dependent patients," Journal of Evolution of Medical and Dental Sciences, vol. 4, no. 53, pp. 9158-9167, 2015.

[2] E. C. Strain, M. R. Lofwall, and J. H. Jaffe, "Opioid related disorders," in Kaplan \& Sadock's Comprehensive Textbook of Psychiatry, B. J. Sadock, V. A. Sadock, and P. Ruiz, Eds., p. 1382, Lippincott Williams \& Wilkins, 9th edition, 2009.

[3] B. P. Smyth, J. Barry, E. Keenan, and K. Ducray, "Lapse and relapse following inpatient treatment of opiate dependence," Irish Medical Journal, vol. 103, no. 6, pp. 176-179, 2010. 
[4] B. P. Bradley, G. Phillips, L. Green, and M. Gossop, "Circumstances surrounding the initial lapse to opiate use following detoxification," British Journal of Psychiatry, vol. 154, pp. 354359, 1989.

[5] B. P. Smyth, J. Barry, A. Lane et al., "In-patient treatment of opiate dependence: medium-term follow-up outcomes," British Journal of Psychiatry, vol. 187, pp. 360-365, 2005.

[6] S. Unnithan, M. Gossop, and J. Strang, "Factors associated with relapse among opiate addicts in an out-patient detoxification programme," British Journal of Psychiatry, vol. 161, pp. 654-657, 1992.

[7] R. Marhe, M. Luijten, and I. H. A. Franken, "The clinical relevance of neurocognitive measures in addiction," Frontiers in Psychiatry, vol. 10, no. 4, article 185, 2014.

[8] B. J. Rounsaville, T. R. Kosten, and H. D. Kleber, "The antecedents and benefits of achieving abstinence in opioid addicts: a 2.5-year follow-up study," The American Journal of Drug and Alcohol Abuse, vol. 13, no. 3, pp. 213-229, 1987.

[9] Y. P. S. Balhara, R. Ranjan, A. Dhawan, and D. Yadav, "Experiences from a community based substance use treatment centre in an urban resettlement colony in India," Journal of Addiction, vol. 2014, Article ID 982028, 6 pages, 2014.

[10] A. Hakansson and E. Hallén, "Predictors of dropout from inpatient opioid detoxification with buprenorphine: a chart review," Journal of Addiction, vol. 2014, Article ID 965267, 5 pages, 2014.

[11] P. Majumder, S. Sarkar, R. Gupta, B. N. Patra, and Y. P. S. Balhara, "Predictors of retention in treatment in a tertiary care de-addiction center," Indian Journal of Psychiatry, vol. 58, no. 1, pp. 27-30, 2016.

[12] E. A. Woodcock, L. H. Lundahl, and M. K. Greenwald, "Predictors of buprenorphine initial outpatient maintenance and dose taper response among non-treatment-seeking heroin dependent volunteers," Drug and Alcohol Dependence, vol. 146, no. 1, pp. 89-96, 2015.

[13] L. Grau-López, C. Roncero, C. Daigre et al., "Risk factors for relapse in drug-dependent patients after hospital detoxification," Adicciones, vol. 24, no. 2, pp. 115-122, 2012.

[14] S. Darke, J. Ross, M. Teesson et al., "Factors associated with 12 months continuous heroin abstinence: findings from the Australian Treatment Outcome Study (ATOS)," Journal of Substance Abuse Treatment, vol. 28, no. 3, pp. 255-263, 2005.

[15] M. Gossop, L. Green, G. Phillips, and B. Bradley, "Factors predicting outcome among opiate addicts after treatment," British Journal of Clinical Psychology, vol. 29, no. 2, pp. 209-216, 1990.

[16] S. M. Singh, S. K. Mattoo, A. Dutt et al., "Long-term outcome of in-patients with substance use disorders: a study from North India," Indian Journal of Psychiatry, vol. 50, no. 4, pp. 269-273, 2008.

[17] B. Broers, F. Giner, P. Dumont, and A. Mino, "Inpatient opiate detoxification in Geneva: follow-up at 1 and 6 months," Drug and Alcohol Dependence, vol. 58, no. 1-2, pp. 85-92, 2000.

[18] A. S. Friedman, A. Terras, and C. Kreisher, "Family and client characteristics as predictors of outpatient treatment outcome for adolescent drug abusers," Journal of Substance Abuse, vol. 7, no. 3, pp. 345-356, 1995.

[19] S. S. Richter, S. A. Brown, and M. A. Mott, "The impact of social support and self-esteem on adolescent substance abuse treatment outcome," Journal of Substance Abuse, vol. 3, no. 4, pp. 371-385, 1991.

[20] T. R. Kosten, B. J. Rounsaville, and H. D. Kleber, "A 2.5 year follow-up of treatment retention and reentry among opioid addicts," Journal of Substance Abuse Treatment, vol. 3, no. 3, pp. 181-189, 1986. 


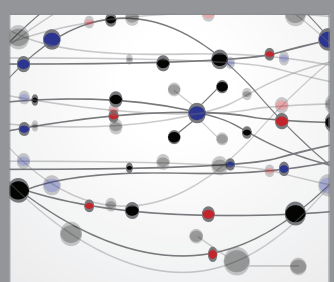

The Scientific World Journal
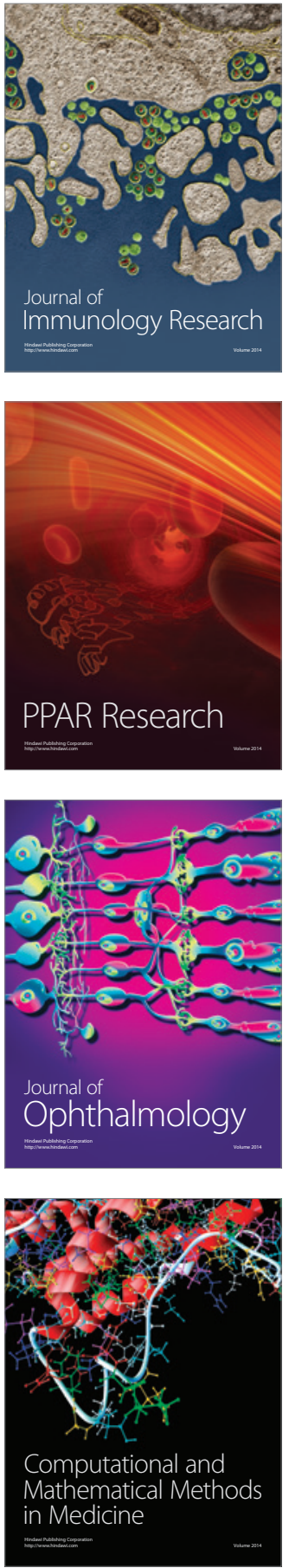

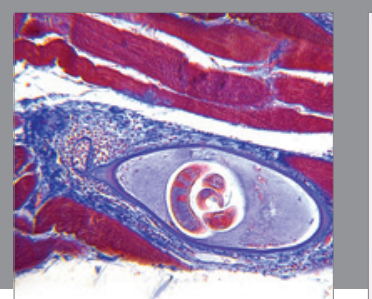

Gastroenterology Research and Practice

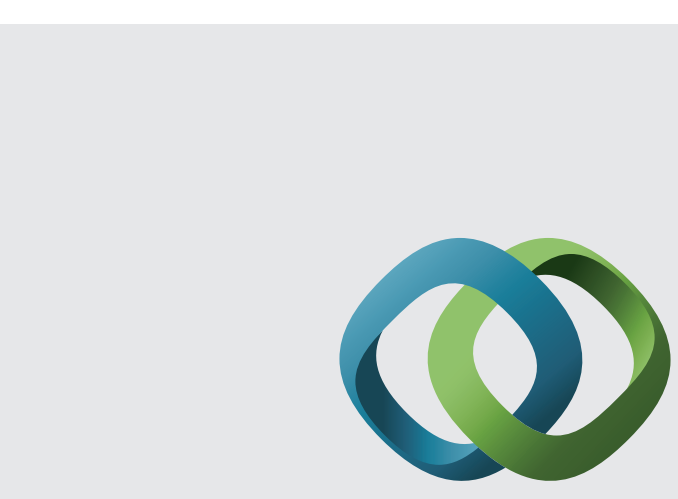

\section{Hindawi}

Submit your manuscripts at

http://www.hindawi.com
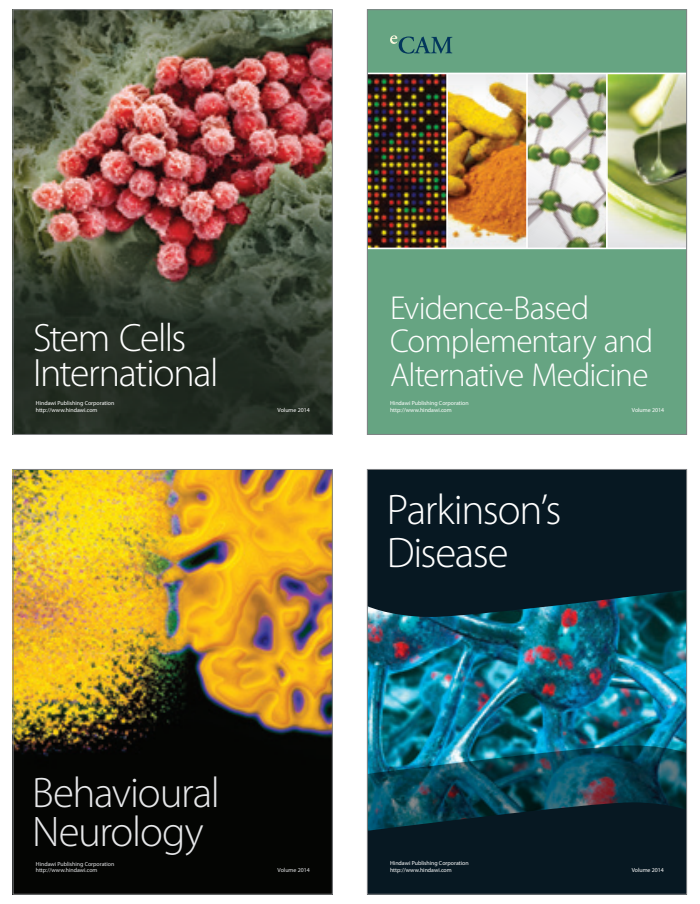
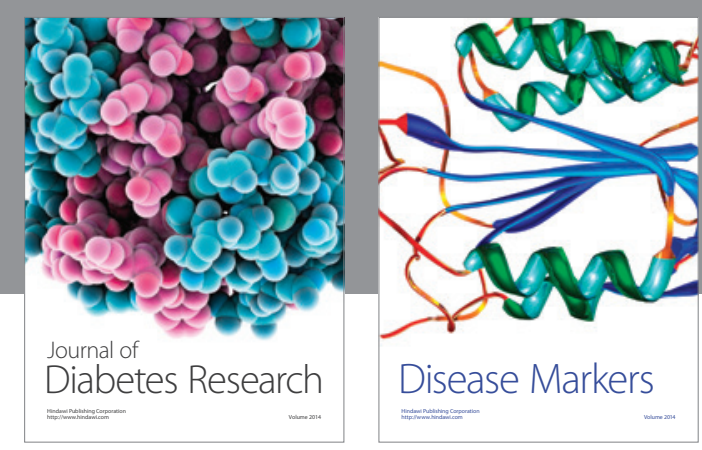

Disease Markers
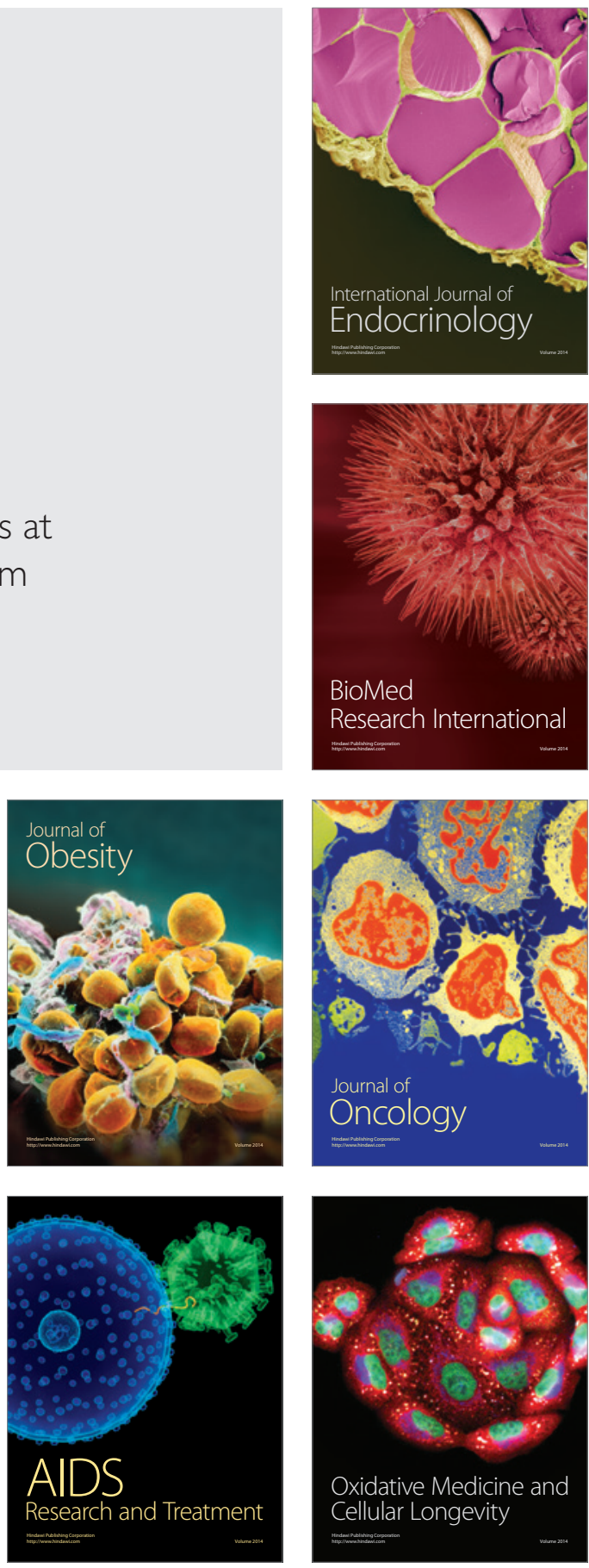